\title{
Retractions of One Dimensional Manifold
}

\author{
A. E. El-Ahmady ${ }^{1}$, Nashwa Salem Awyd Al-Hazmi ${ }^{2}$ \\ ${ }^{1}$ Mathematics Department, Faculty of Science, Taibah University, Madinah, KSA \\ ${ }^{2}$ Mathematics Department, Faculty of Science, Tanta University, Tanta, Egypt \\ Email: hk.mishra@juet.ac.in
}

Received July 23, 2012; revised September 10, 2012; accepted September 17, 2012

\begin{abstract}
Our aim in the present article is to introduce and study types of retraction of one dimensional manifold. New types of geodesics in one dimensional manifold are presented. The deformation retracts of one dimensional manifold into itself and onto geodesics is deduced. Also, the isometric and topological folding in each case and the relation between the deformations retracts after and before folding has been obtained. New types of conditional folding are described.
\end{abstract}

Keywords: Retractions; Deformation Retracts; Folding; One Dimensional Manifold

\section{Introduction}

As is well known, the theory of retractions is always one of interesting topics in Euclidian and Non-Euclidian space and it has been investigated from the various viewpoints by many branches of topology and differential geometry El-Ahmady [1-3].

Most folding problems are attractive from a pure mathematical standpoint, for the beauty of the problems themselves. The folding problems have close connections to important industrial applications Linkage folding has applications in robotics and hydraulic tube bending. Paper folding has application in sheet-metal bending, packaging, and air-bag folding El-Ahmady [2,3]. Following the great Soviet geometer El-Ahmady [4], also used folding to solve difficult problems related to shell structures in civil engineering and aero space design, namely buckling instability El-Ahmady [4].

Isometric folding between two Riemannian manifold may be characterized as maps that send piecewise geodesic segments to a piecewise geodesic segments of the same length El-Ahmady [2-4]. For a topological folding the maps do not preserves lengths El-Ahmady $[5,6]$, i.e. A map $\mathfrak{I}: M \rightarrow \mathrm{N}$, where $M$ and $N$ are $C^{\infty}$-Riemannian manifolds of dimension $\mathrm{m}, \mathrm{n}$ respectively is said to be an isometric folding of $M$ into $N$, iff for any piecewise geodesic path $\gamma: J \rightarrow M$, the induced path $\mathfrak{I} \circ \gamma: J \rightarrow \mathrm{N}$ is a piecewise geodesic and of the same length as $\gamma$, If $\mathfrak{I}$ does not preserve length, then $\mathfrak{I}$ is a topological folding El-Ahmady $[7,8]$.

A subset $A$ of a topological space $X$ is called a retract of $X$ if there exists a continuous map $r: X \rightarrow A$ such that $r(a)=a, \forall a \in A$ where $A$ is closed and $X$ is open El-Ahmady [9-11]. Also, let $X$ be a space and $A$ a subspace. A map $r: X \rightarrow A$ such that $r(a)=a$, for all $a \in A$, is called a retraction of $\mathrm{X}$ onto $\mathrm{A}$ and $\mathrm{A}$ is called a retract of $X$. This can be restated as follows. If $i: A \rightarrow X$ is the inclusion map, then $r: X \rightarrow A$ is a map such that $r i=i d_{A}$ If, in addition, $r i \simeq i d_{X}$, we call $r$ a deformation retract and $A$ a deformation retract of $X$ Another simple-but extremely useful-idea is that of a retract. If $A, X \subset M$ then $A$ is a retract of $X$ if there is a commutative diagram.

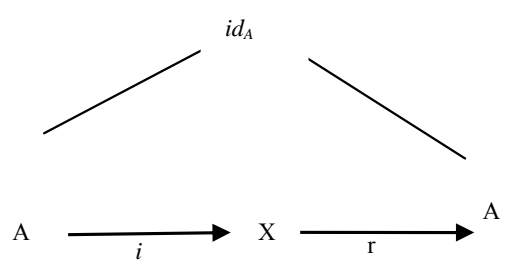

If $f: A \rightarrow B$ and $g: X \rightarrow Y$, then $f$ is a retract of $g$ if there is a commutative diagram El-Ahmady [3,7], Arkowitz [12], Naber [13,14], Reid [15], Shick [16] and Strom [17].

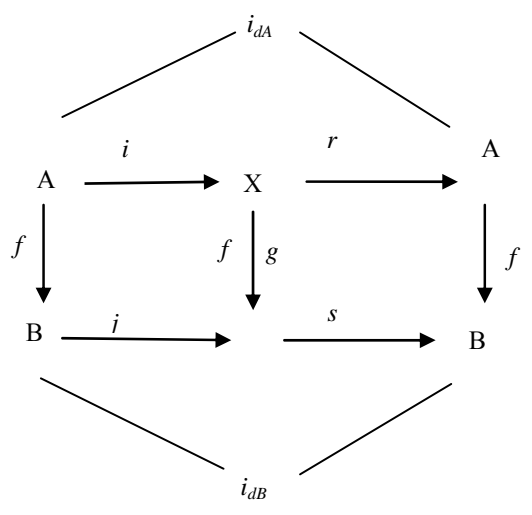




\section{Main Result}

\subsection{On a Closed Interval}

In what follows, we discuss the retractions, let the closed interval be $I=[a, b]$, since the closed interval $I$ is closed then firstly take a point $\{p\}$ of it to make a retraction, $([a, b]-\{p\})$ is open. Consider some types of retractions of $([a, b]-\{p\})$.

$$
\text { If } r_{i}:([a, b]-\{p\}) \rightarrow C^{*}, C^{*} \subset I
$$

then we can get

$$
\begin{aligned}
& \left.\left.r_{1}([a, b]-\{p\})=\right] a, b\right], r_{2}([a, b]-\{p\})=[a, b[ \\
& \left.r_{3}([a, b]-\{p\})=\right] a, b\left[, r_{4}([a, b]-\{p\})=0,0\right.
\end{aligned} .
$$

Now, we are in a position to formulate the following two theorems.

Theorem 1. All types of retraction of a closed interval are semi-open set or open set or zero-space.

Theorem 2. The limit of retraction of closed interval is a zero-manifold.

Now, we are going to discuss the deformation retract of the closed interval. Let $([a, b]-\{p\})$ be the open interval, and then the homotopy map is defined as $\eta_{i}:([a, b]-\{p\}) \times I \rightarrow([a, b]-\{p\})$, where $I=[0,1]$, then we present the following cases of deformation retracts

$$
\left.\left.\eta_{1}(x, t)=(1-t)([a, b]-\{p\})+t(] a, b\right]\right),
$$

where $\eta_{1}(x, 0)=([a, b]-\{p\})$

and

$$
\begin{aligned}
& \left.\left.\eta_{1}(x, 1)=\right] a, b\right] \\
& \eta_{2}(x, t)=e^{t}(1-t)([a, b]-\{p\})+\frac{1}{2} t(2 t+t)([a, b[), \\
& \eta_{3}(x, t)=\cos \frac{\pi}{2} t([a, b]-\{p\})+\sin \frac{\pi}{2} t(] a, b[), \\
& \eta_{4}(x, t)=\frac{1-t}{1+t}([a, b]-\{p\})+\frac{2 t}{1+t}\left\{v^{i}, i=1,2\right\}
\end{aligned}
$$

From the above discussion, we obtain the following theorem.

Theorem 3. The deformation retracts of a closed interval gives semi-open set, open set and zero-dimensional space.

Now, we are going to discuss the folding $g$ of closed interval $I$.

Let $g: I \rightarrow I$, where $g(x)=|x|$.

An isometric folding of closed interval $I$ into itself may by

$$
g:([a, b]-\{p\}) \rightarrow|([a, b]-\{p\})|
$$

The deformation retracts of the folded closed interval $g(I)$ into the folded retraction $g\left(r_{1}\right)$ is

$$
\eta g:|([a, b]-\{p\})| \times I \rightarrow|([a, b]-\{p\})|,
$$

with $\left.\left.\eta g(m, c)=\cos \frac{\pi c}{2}|([a, b]-\{p\})|+\sin \frac{\pi c}{2}(\mid] a, b\right] \mid\right)$,

where $\eta g(m, 0)=|([a, b]-\{p\})|$

and $\eta g(m, 1)=(\mid] a, b] \mid)$

The deformation retract of the folded closed interval $g(I)$ into the folded retraction $g\left(r_{4}\right)$ is

$$
\eta g(m, c)=\cos \frac{\pi c}{2}|([a, b]-\{p\})|+\sin \frac{\pi c}{2}(\mid[a, b[\mid)
$$

The deformation retract of the folded closed interval $g(I)$ into the folded retraction $g\left(r_{4}\right)$ is

$$
\eta g(m, c)=e^{c}(1-c)|([a, b]-\{p\})|+\frac{1}{2} c(2 c+c)(0,0)
$$

Then, the following theorem has been proved.

Theorem 4. The deformation retract of the folded closed interval into the folded retractions is the same as or different from the deformation retract of the closed interval into the retractions

Proposition 1. If the retraction of the closed interval $[a, b]$ is $r:([a, b]-\{p\}) \rightarrow c^{*}$ and the folding of $([a, b]-\{p\})$ into itself is

$f:([a, b]-\{p\}) \rightarrow|([a, b]-\{p\})|$, then there are commutative diagram between retraction and folding such that

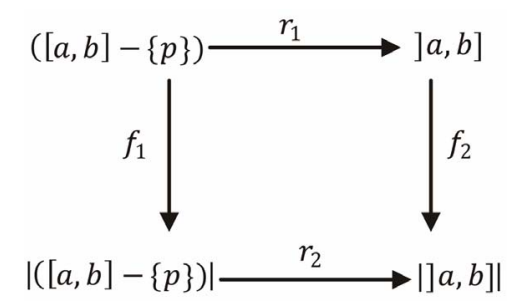

Proof. Let a retraction $\left.\left.r_{1}:([a, b]-\{p\}) \rightarrow\right] a, b\right]$, be a retraction of $([a, b]-\{p\})$ into $] a, b]$. Also, let the folding $\quad f_{1}$ is $f_{1}:([a, b]-\{p\}) \rightarrow|([a, b]-\{p\})|$ and the folding $f_{2}$ be $\left.\left.\left.\left.f_{2}:(] a, b\right]\right) \rightarrow \mid\right] a, b\right] \mid$. Then we have the retraction $\left.\left.r_{2}:|([a, b]-\{p\})| \rightarrow \mid\right] a, b\right] \mid$ such that

$$
f_{2}^{\circ} r_{1}=r_{2}^{\circ} f_{1}
$$

Proposition 2. The relation between the retraction of the closed interval and the limit of folding discussed from the following commutative diagram. 


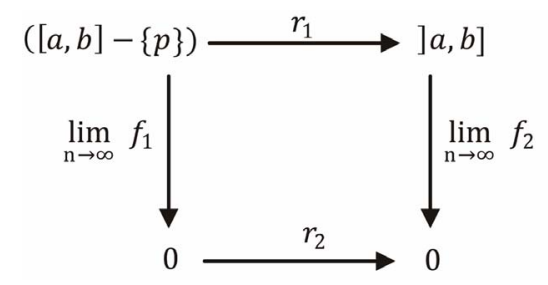

Proof. Let a retraction $\left.\left.r_{1}:([a, b]-\{p\}) \rightarrow\right] a, b\right]$, be a retraction of $([a, b]-\{p\})$ into $] a, b]$. Also, let the limits of folding are given by

$\lim _{\mathrm{n} \rightarrow \infty} f_{1}:([a, b]-\{p\}) \rightarrow 0$ and $\left.\left.\lim _{\mathrm{n} \rightarrow \infty} f_{2}:\right] a, b\right] \rightarrow 0$.

Then we have the retraction of the zero dimensional manifold is the identity map, i.e. $r_{2}: 0 \rightarrow 0$ such that

$$
\lim _{n \rightarrow \infty} f_{2}{ }^{\circ} r_{1}=r_{2}{ }^{\circ} \lim _{n \rightarrow \infty} f_{1}
$$

\subsection{On a Cartesian Product of Closed Interval}

In this position, we introduce the retraction of Cartesian product of closed interval. Consider two closed intervals $I_{1}=I_{2}=[0,1]$. The Cartesian product is defined as

$$
I^{2}=[0,1] \times[0,1]=\{(0,0),(0,1)(1,0)(1,1)\} .
$$

The retraction is defined as. Consider the square $I^{2}$ with vertices $v^{i} ; i=0,1,2,3$, removing only one vertex $v^{i} ; i=0,1,2,3$, then the retraction is given by,

$$
r_{1}:\left(I \times I-\left\{v^{i} ; i=0,1,2,3\right\}\right) \rightarrow \text { Adjacent edges . }
$$

Also, removing two adjacent vertices is equivalent to removing an edge of the square, and then the retraction is defined as follows,

$$
r_{2}:\left(I \times I-\left\{v^{i}, i=0 \text { and } 1\right\}\right) \rightarrow \text { closed interva. }
$$

Moreover, removing two non-adjacent vertices gives a retraction, which is directly the zero-dimensional manifold,

$$
r_{3}:\left(I \times I-\left\{v^{i}, i=1 \text { and } 3\right\}\right) \rightarrow \text { zero-points }
$$

In what follows, we discuss the deformation retract of the square $I^{2}$ as follows. The deformation retract of the square $I^{2} \subset R^{2}$ is defined by

$$
\begin{aligned}
& \eta_{j}:\left(I^{2}-\left\{v^{i} ; i=0,1,2,3\right\}\right) \times I \\
& \rightarrow\left(I^{2}-\left\{v^{i} ; i=0,1,2,3\right\}\right),
\end{aligned}
$$

where $I$ is the closed interval $[0,1]$. Then we have the following cases of deformation retract. The deformation retract of the square $I^{2} \subset R^{2}$ onto a adjacent edges $\subset I^{2}$ is given by

$$
\begin{aligned}
& \eta_{1}(x, t) \cos \frac{\pi}{2} t\left(I^{2}-\left\{v^{i} ; i=0,1,2,3\right\}\right) \\
& +\sin \frac{\pi}{2} t(\text { adjacent edges })
\end{aligned}
$$

where $\eta_{1}(x, 0)=\left(I^{2}-\left\{v^{i} ; i=0,1,2,3\right\}\right)$,

and $\eta_{1}(x, 1)=$ adjacent edges.

The deformation retract of the square $I^{2} \subset R^{2}$ onto closed interval $I$ will be

$$
\eta_{2}(x, t)=(1-t)\left(I^{2}-\left\{v^{i} ; i=0,1,2,3\right\}\right)+t I
$$

The deformation retract of the square $I^{2} \subset R^{2}$ onto zero-points is

$$
\begin{aligned}
\eta_{3}(x, t)= & e^{t}(1-t)\left(I^{2}-\left\{v^{i} ; i=0,1,2,3\right\}\right) \\
& +\frac{1}{2} t\left(2 t+\frac{1}{2}\right) \text { (zero points). }
\end{aligned}
$$

From all the above discussion, we arrive to the following theorem.

Theorem 5. The limit of retractions sequence of the square is the 0 -dimensional manifold. Also, the deformation retract of the square is either subsquare or zero-dimensional manifold.

Proposition 3. If the retraction of the square $I^{2} \subset \mathbb{R}^{2}$ is

$r:\left(I^{2}-\left\{v^{i} ; i=0,1,2,3\right\}\right) \rightarrow$ Adjacent edges, $I$, zero-point.

and the folding of $\left(I^{2}-\left\{v^{i} ; i=0,1,2,3\right\}\right)$ into itself is

$$
f:\left(I^{2}-\left\{v^{i} ; i=0,1,2,3\right\}\right) \rightarrow\left(I^{2}-\left\{v^{i} ; i=0,1,2,3\right\}\right) \text {. }
$$

Then there are commutative diagrams between retractions and foldings such that

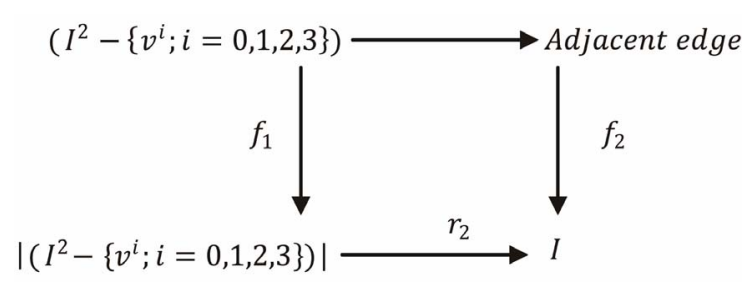

Also

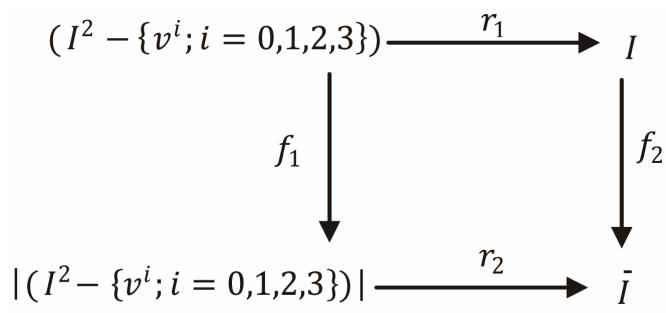

And also 


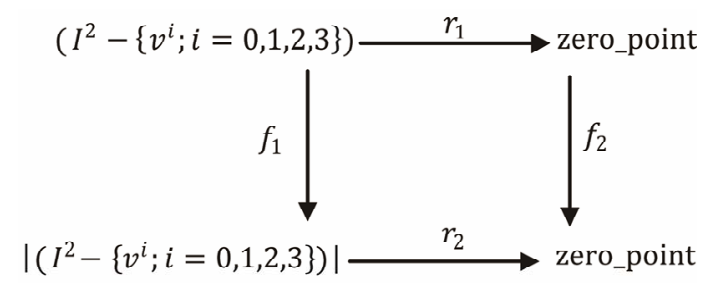

Proposition 4. The relation between the retraction of the square $I^{2} \subset \mathbb{R}^{2}$ and the limit of folding discussed from the following commutative diagrams.

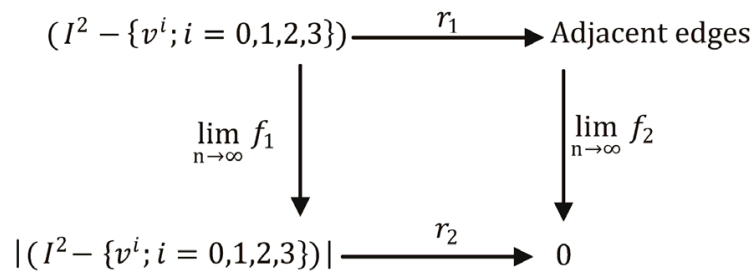

Also

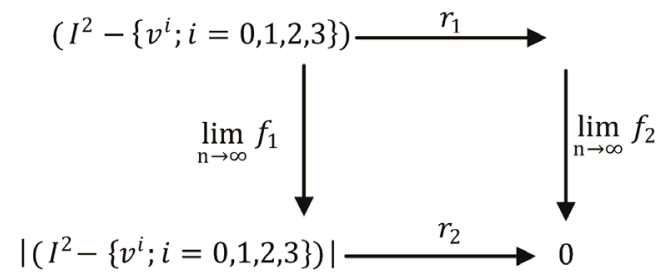

Again

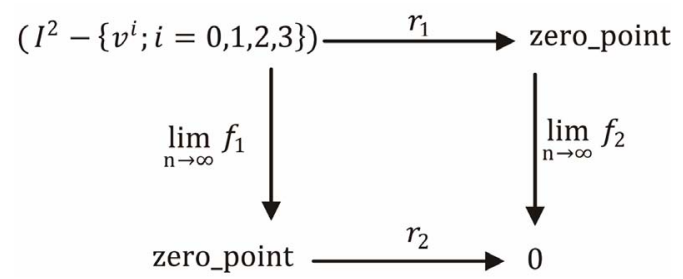

where the limit of the folding of the Cartesian product of $I^{2} \subset \mathbb{R}^{2}$ is not equal to the Cartesian product of their limits.

Proposition 5. If the deformation retract of $I^{2} \subset \mathbb{R}^{2}$ $D:\left(I^{2}-\left\{v^{i} ; i=0,1,2,3\right\}\right) \times I \rightarrow\left(I^{2}-\left\{v^{i} ; i=0,1,2,3\right\}\right)$, where $I \in[0,1]$ and the retraction of $I^{2} \subset \mathbb{R}^{2}$ is $r:\left(I^{2}-\left\{v^{i} ; i=0,1,2,3\right\}\right) \rightarrow$ Adjacent edges, zero-point and the folding of $I^{2} \subset \mathbb{R}^{2}$ into itself is $\mathrm{f}: I^{2} \subset \mathbb{R}^{2} \rightarrow I^{2} \subset \mathbb{R}^{2}$. Then there are induces deformation retractions, and folding such that the following diagram is commutative.

Proof. Let the deformation retract of $I^{2} \subset \mathbb{R}^{2}$ is $D_{1}:\left(I^{2}-\left\{v^{i} ; i=0,1,2,3\right\}\right) \times I \rightarrow\left(I^{2}-\left\{v^{i} ; i=0,1,2,3\right\}\right)$, the folding of $I^{2} \times I$, and
$D_{1}\left(\left(I^{2}-\left\{v^{i} ; i=0,1,2,3\right\}\right) \times I \rightarrow\left(I^{2}-\left\{v^{i} ; i=0,1,2,3\right\}\right)\right)$ are defined by $f_{1}: I^{2} \times I \rightarrow I^{2} \times I$ also $f_{2}: D_{1}\left(I^{2}-\left\{v^{i} ; i=0,1,2,3\right\}\right) \times I \rightarrow$ Adjacent edges, the deformation retract of $\mathrm{f}_{1}\left(I^{2} \times I\right)$ is $D_{2}: \mathrm{f}_{1}\left(I^{2} \times I\right) \rightarrow$ Adjacent edges and the retractions of $\left(f_{2}\left(D_{1}\left(I^{2}-\left\{v^{i} ; i=0,1,2,3\right\}\right) \times I\right)\right)$, and $D_{2}\left(f_{1}\left(I^{2} \times I\right)\right)$ are given by $r_{1}: f_{2}\left(D_{1}\left(I^{2}-\left\{v^{i} ; i=0,1,2,3\right\}\right)\right) \times I \rightarrow I$, $r_{2}: D_{2}\left(f_{1}\left(I^{2} \times I\right)\right) \rightarrow I$. Hence, the following diagram is commutative

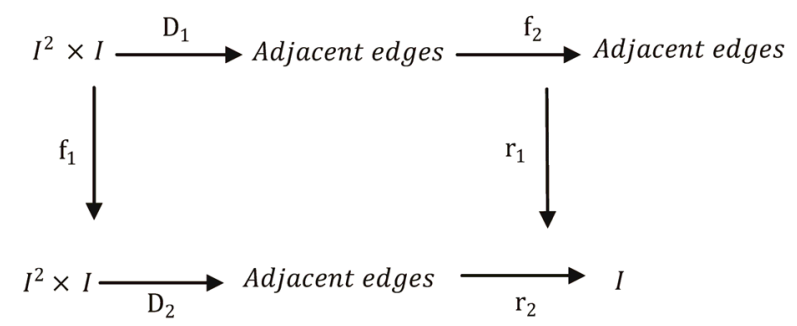

Also, the end of limits of the folding and the end of limits of retractions of $I^{2} \subset \mathbb{R}^{2}$ induces the 0-dimensional space which is a point and in this case the retraction and folding of 0-dimensional space coincide.

Proposition 6. The limit of the folding of 0-dimensional space $M$ is 0 -dimensional space.

Proof. Let $M$ be an $n$-dimensional space, consider the limit of the folding $\lim _{m \rightarrow \infty} f_{m}: M \rightarrow \bar{M}$,

$\lim _{m \rightarrow \infty} f_{m}(M) \subset M$, then $\operatorname{dim}\left(\lim _{m \rightarrow \infty} f_{m}(M)\right) \leq \operatorname{dim}(M)$,

but if $M$ has 0 -dimension. Then $\operatorname{dim}\left(\lim _{m \rightarrow \infty} f_{m}(M)\right) \leq 0$.

Since $\operatorname{dim} \varnothing=-1$, then $\operatorname{dim}\left(\lim _{m \rightarrow \infty} f_{m}(M)\right)=0$.

Theorem 6. $\left(I_{1}^{2}, x_{0}\right)$ is a strong deformation retract of $\left(I^{2}, x_{0}\right)$.

Proof. Let $D:\left(I^{2}-(0,0)\right) \times[0, m] \rightarrow\left(I^{2}-(0,0)\right)$, where $I^{2} \subset \mathbb{R}^{2}, \quad m \in Z$ is a strong deformation retracting of $\left(I^{2}, x_{0}\right), x_{0}=(0,0)$ into $I_{1}^{2}$. To be specific, the k-homotopy $\mathrm{D}$ is assumed:

$D(x, 0)=x$ for all $x \in I^{2}, D(x, m) \in I_{1}^{2}$ for all $x \in I^{2}$, and $D(a, t)=\mathrm{a}$ for all $a \in I_{1}^{2}$, and for all $t \in[0, m]$.

Let $r:\left(I^{2}-(0,0)\right) \rightarrow I_{1}^{2}$, be defined as

$$
\begin{aligned}
& \{(x, y):-10 \leq x \leq 10,:-10 \leq y \leq 10\} \rightarrow \\
& \{(x, y):-1<x<5,: 2<y<-2\}, i: I_{1}^{2} \rightarrow I^{2} \\
& \quad(\mathrm{i} \circ r)(x)=D(x, m)=x \text { for all } x \in I^{2},
\end{aligned}
$$

Also $(\mathrm{r} \circ i)=\mathrm{I}_{I_{1}^{2}, x_{0}}$. 
Then, $\left(I_{1}^{2}, x_{0}\right)$ is a strong deformation retract of $\left(I^{2}, x_{0}\right)$.

Proposition 7. The retraction of $\left(I^{2}-(0,0)\right)$ is a two-dimensional manifold and the limit of foldings is a one-manifold.

Proof. If $\mathrm{A}$ is a retraction of $\left(I^{2}-(0,0)\right)$, then either dimension $\mathrm{A}=$ dimension $\left(I^{2}-(0,0)\right)$ or dimension $\mathrm{A}$ $\neq$ dimension $\left(I^{2}-(0,0)\right)$, but in this case dimension $\left(I^{2}-(0,0)\right)$ not invariant. Then $\left(I^{2}-(0,0)\right)$ is the same dimension of $I^{2}$. But, the limit of the foldings of 2-dimensional manifold into itself is a manifold of dimension $n-1$. Then, the limit of foldings is a one-manifold.

\subsection{On a Circle}

Theorem 7. If $I^{2} \subset \mathbb{R}^{2}$ has the fixed point property, then $S^{1}$ is not a retract of $I^{2}$.

Proof. Let $I^{2} \subset \mathbb{R}^{2}$ has the fixed point property. Observe that $S^{1}$ certainly does not have the fixed point property since, for example, the antipodal map- $\mathrm{id}_{\mathrm{s}^{1}}$ is continuous, but has no fixed points. Then $S^{1}$ can therefore not be a retract of $I^{2}$.

Proposition 8. If $I^{2} \subset \mathbb{R}^{2}$ has the fixed point property, then $r\left(S^{1}-m\right)$ is not a retract of $I^{2}$, where $\operatorname{dim}\left(r\left(S^{1}-m\right)\right)=1, m \in S^{1}$.

Proof. Since $r\left(S^{1}-m\right) \subset S^{1}$, and $S^{1}$ does not have the fixed point property. Then $r\left(S^{1}-m\right)$ does not have the fixed point property, $\operatorname{dim}\left(r\left(S^{1}-m\right)\right)=1$. Then $r\left(S^{1}-m\right)$ is not a retract of $I^{2}$.

Theorem 8. If $S^{0}$ is a 0 -manifold, then $S^{0}$ is a retract of $\left(S^{1^{*}}-n\right)$, such that $S^{0} \subset\left(S^{1^{*}}-n\right)$, $\left(S^{1^{*}}-n\right)=r\left(S^{1}-m\right)$, and $\operatorname{dim}\left(S^{1^{*}}-n\right)=\operatorname{dim}\left(S^{1}-m\right)$, where $m \in S^{1}$ and $n \in S^{1^{*}}$.

Proof. Now, let $r_{i}:\left(S^{1}-m\right) \rightarrow S^{1^{*}}, S^{1^{*}} \subset\left(S^{1}-m\right)$, $m \in S^{1}$ be the retraction map of $S^{1}$ defined as $r_{1}\left(S^{1}-m\right)=q\left(\cos t^{*}, \sin t^{*}\right), \pi<t^{*}<\frac{3 \pi}{2}$. Let the inclusion map of $S^{0}$, where $S^{0}=q(0,1)$, is $i_{1}: S^{0} \rightarrow\left(S^{1}-m\right)$, the retractions of $S^{0}$ and $i_{1}\left(S^{0}\right)$ are defined by $r_{1}: S^{0} \rightarrow S^{0}$ and $r_{2}: i_{1}\left(S^{0}\right) \rightarrow\left(S^{1^{*}}-n\right)$, where $S^{1^{*}}=q\left(\cos t^{*}, \sin t^{*}\right), 0<t^{*} \leq \frac{\pi}{2}$,

$r_{2}: i_{1}\left(S^{0}\right) \rightarrow\left(S^{1^{*}}-n\right)$ is the inclusion map of $r_{1}\left(S^{0}\right)$, the retraction of $i_{1}\left(S^{0}\right)$ is $r_{3}: i_{1}\left(S^{0}\right) \rightarrow S^{0}$, also the retractions of $r_{3}\left(i_{1}\left(S^{0}\right)\right)$ and $i_{2}\left(r_{1}\left(S^{0}\right)\right)$ are given by $r_{4}: r_{3}\left(i_{1}\left(S^{0}\right)\right) \rightarrow S^{0}$ and $r_{5}: i_{2}\left(r_{1}\left(S^{0}\right)\right) \rightarrow S^{0}$. Hence, the following diagram is commutative:

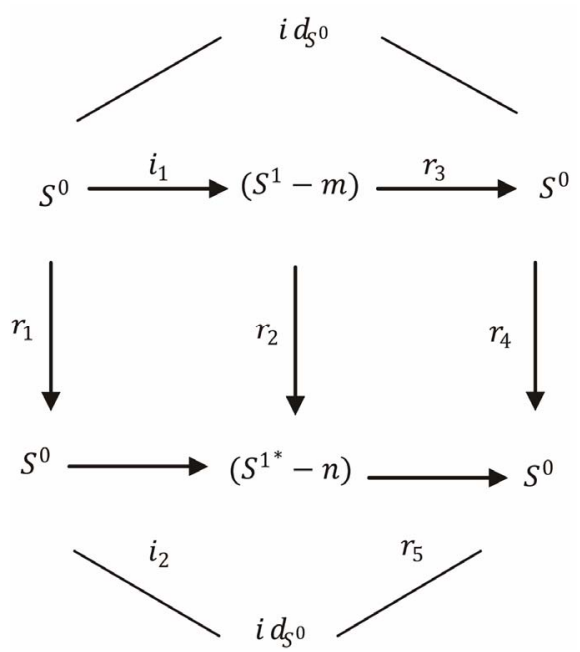

Proposition 9. The relation between the retraction, the limit of the folding and the inclusion map of circle $S^{1}$ discussed from the following commutative diagram

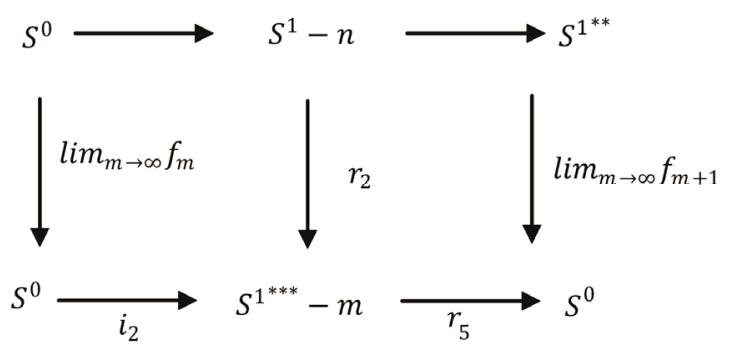

where

$$
\begin{aligned}
& S^{1^{*}}=q\left(\cos t^{*}, \sin t^{*}\right), 0 \leq t^{*}<\frac{3 \pi}{2}, \\
& S^{1 * *}=q\left(\cos t^{* *}, \sin t^{* *}\right), 0 \leq t^{* *}<\pi .
\end{aligned}
$$

and

$$
S^{1 * * *}=q\left(\cos t^{* * *}, \sin t^{* * *}\right), 0 \leq t^{* * *}<\frac{\pi}{2}, n \in S^{1}, m \in S^{1 * * *}
$$

The purpose of this position is to introduce the relation between the deformation retract and folding of the circle, the parametric equation of the open circle in the plane is given by

$$
S^{1}=((a \cos t, a \sin t)-\{p\}) .
$$

Now consider some types of retractions of the circle $S^{1}=((a \cos t, a \sin t)-\{p\})$, if

$$
r_{i}: S^{1}=((a \cos t, a \sin t)-\{p\}) \rightarrow S^{*}, S^{*} \subset S^{1},
$$

then we can get

$$
r_{1}((a \cos t, a \sin t)-\{p\})=(a, 0), t=0,
$$




$$
\begin{aligned}
& r_{2}((a \cos t, a \sin t)-\{p\})=\left(\frac{a \sqrt{3}}{2}, \frac{a}{2}\right), t=\frac{\pi}{6}, \\
& r_{3}((a \cos t, a \sin t)-\{p\})=\left(\frac{a \sqrt{2}}{2}, \frac{a \sqrt{2}}{2}\right), t=\frac{\pi}{4}, \\
& r_{4}((a \cos t, a \sin t)-\{p\})=\left(\frac{a}{2}, \frac{a \sqrt{3}}{2}\right), t=\frac{\pi}{3}, \\
& r_{5}((a \cos t, a \sin t)-\{p\})=(0, a), t=\frac{\pi}{2}, \\
& r_{6}((a \cos t, a \sin t)-\{p\})=\left(\frac{-a}{2}, \frac{a \sqrt{3}}{2}\right), t=\frac{2 \pi}{3}, \\
& r_{7}((a \cos t, a \sin t)-\{p\})=\left(\frac{-a \sqrt{2}}{2}, \frac{a \sqrt{2}}{2}\right), t=\frac{3 \pi}{4}, \\
& r_{8}((a \cos t, a \sin t)-\{p\})=\left(\frac{-a \sqrt{3}}{2}, \frac{a}{2}\right), t=\frac{5 \pi}{6}, \\
& r_{9}((a \cos t, a \sin t)-\{p\})=(-a, 0), t=\pi, \\
& r_{17}\left(((a \cos t, a \sin t)-\{p\})=\left(\frac{-a \sqrt{3}}{2}, \frac{-a}{2}\right), t=\frac{7 \pi}{6},\right. \\
& r_{16}((a \cos t, a \sin t)-\{p\})=\left(\frac{a \sqrt{3}}{2}, \frac{-a}{2}\right), t=\frac{11 \pi}{6}, \\
& r_{13}((a \cos t, a \sin t)-\{p\})=(0,-a), t=\frac{3 \pi}{2}, \\
& r_{14}((a \cos t, a \sin t)-\{p\})=\left(\frac{a}{2}, \frac{-a \sqrt{3}}{2}\right), t=\frac{5 \pi}{3}, \\
& r_{11}((a \cos t, a \sin t)-\{p\})=\left(\frac{-a \sqrt{2}}{2}, \frac{-a \sqrt{2}}{2}\right), t=\frac{5 \pi}{4}, \\
& r_{12}((a \cos t, a \sin t)-\{p\})=\left(\frac{-a}{2}, \frac{-a \sqrt{3}}{2}\right), t=\frac{4 \pi}{3},
\end{aligned}
$$

Now, we are going to discuss the deformation retract of the circle. Let $S^{1}=((a \cos t, a \sin t)-\{p\})$ be the open circle, then the homotopy map is defined as $\eta:((a \cos t, a \sin t)-\{p\}) \times I \rightarrow((a \cos t, a \sin t)-\{p\})$, where $I=[0,1]$, Then we have the following cases of deformation retracts.

$$
\eta_{1}(x, t)=(1-t)((a \cos t, a \sin t)-\{p\})+t(a, 0),
$$

where $\eta_{1}(x, 0)=((a \cos t, a \sin t)-\{p\})$ and $\eta_{1}(x, 1)=(a, 0)$,

$$
\begin{aligned}
\eta_{2}(x, t)= & e^{t}(1-t)((a \cos t, a \sin t)-\{p\}) \\
& +\frac{1}{2} t(2 t+t)\left(\frac{a \sqrt{3}}{2}, \frac{a}{2}\right), \\
\eta_{3}(x, t)= & \cos \frac{\pi t}{2}((a \cos t, a \sin t)-\{p\}) \\
& +\sin \frac{\pi t}{2}\left(\frac{a \sqrt{2}}{2}, \frac{a \sqrt{2}}{2}\right) \\
\eta_{4}(x, t)= & \frac{1-t}{1+t}((a \cos t, a \sin t)-\{p\})+\frac{2 t}{1+t}\left(\frac{a}{2}, \frac{a \sqrt{3}}{2}\right), \\
\eta_{5}(x, t)= & \frac{1-t}{1+t}((a \cos t, a \sin t)-\{p\})+\frac{2 t}{1+t}(0, a), \\
\eta_{6}(x, t)= & e^{t}(1-t)((a \cos t, a \sin t)-\{p\}) \\
& +\frac{1}{2} t(2 t+t)\left(\frac{-a}{2}, \frac{a \sqrt{3}}{2}\right),
\end{aligned}
$$$$
\eta_{7}(x, t)=\cos \frac{\pi t}{2}((a \cos t, a \sin t)-\{p\})
$$$$
+\sin \frac{\pi t}{2}\left(\frac{-a \sqrt{2}}{2}, \frac{a \sqrt{2}}{2}\right),
$$$$
\eta_{8}(x, t)=\frac{1-t}{1+t}((a \cos t, a \sin t)-\{p\})
$$$$
+\frac{2 t}{1+t}\left(\frac{-a \sqrt{3}}{2}, \frac{a}{2}\right)
$$

$$
\begin{aligned}
\eta_{9}(x, t)= & \cos \frac{\pi t}{2}((a \cos t, a \sin t)-\{p\}) \\
& +\sin \frac{\pi t}{2}(-a, 0) .
\end{aligned}
$$

Now, we are going to discuss the folding $g$ of the circle $S^{1}$. let $g: S^{1} \rightarrow S^{1}$, where $g\left(x_{1}, x_{2}\right)=\left(x_{1},\left|x_{2}\right|\right)$. An isometric folding of the circle $S^{1}$ into itself may by defined by

$$
g:((a \cos t, a \sin t)-\{p\}) \rightarrow((a \cos t,|a \sin t|)-\{p\})
$$

The deformation retracts of the folded circle $g\left(S^{1}\right)$ into the folded retraction $g\left(\eta_{1}(x, 1)\right)$ is

$$
\begin{aligned}
& \eta g:((a \cos t,|a \sin t|)-\{p\} \times I) \\
& \rightarrow((a \cos t,|a \sin t|)-\{p\})
\end{aligned}
$$

with 


$$
\begin{aligned}
\eta_{1} g(m, t) & =\cos \frac{\pi t}{2}((a \cos t,|a \sin t|)-\{p\}) \\
& +\sin \frac{\pi t}{2}(a, 0)
\end{aligned}
$$

where

$$
\eta_{1} g(m, 0)=((a \cos t,|a \sin t|)-\{p\})
$$

and $\eta_{1} g(m, 1)=(a, 0)$

Then, the following theorem has been proved.

Theorem 9. Under the defined folding and any folding homeomorphic to this type of folding, the deformation retracts of the folded circle into the folded retractions is the same as the deformation retracts of the circle into the retractions.

If the folding be defined by $\mathfrak{I}\left(x_{1}, x_{2}\right)=\left(\left|x_{1}\right|, x_{2}\right)$.

The isometric folded of the circle $S^{1}$ is defined as $\mathfrak{I}((a \cos t, a \sin t)-\{p\}=((|a \cos t|, a \sin t)-\{p\})$

The deformation retract of the folded circle $\mathfrak{I}\left(S^{1}\right)$ into the folded retraction $\mathfrak{I}\left(\eta_{6}(x, t)\right)$ is

$$
\begin{aligned}
\eta \mathfrak{I} & :(|a \cos t|, a \sin t)-\{p\} \times I \\
& \rightarrow((|a \cos t|, a \sin t)-\{p\})
\end{aligned}
$$

with

$$
\begin{aligned}
\eta_{6} \mathfrak{I}(m, t)= & e^{t}(1-t)(|a \cos t|, a \sin t) \\
& -\{p\}+\frac{1}{2} t(2 t+t)\left(\left|\frac{a}{2}\right|, \frac{a \sqrt{3}}{2}\right)
\end{aligned}
$$

The deformation retract of the folded circle $\mathfrak{I}\left(S^{1}\right)$ into the folded retraction $\mathfrak{J}\left(\eta_{8}(x, t)\right)$ is

$$
\begin{aligned}
\eta_{8} \mathfrak{I}(x, t)= & \frac{1-t}{1+t}((|a \cos t|, a \sin t)-\{p\}) \\
& +\frac{2 t}{1+t}\left(\left|\frac{a \sqrt{3}}{2}\right|, \frac{a}{2}\right)
\end{aligned}
$$

Then, the following theorem has been proved.

Theorem 10. Under the defined folding and any folding homeomorphic to this type of folding, the deformation retract of the folded circle into the folded retractions is different from the deformation retract of the circle into the retractions.

Proposition 10. If the retraction of the circle $S^{1} \subset \mathbb{R}^{2}$ is: $((a \cos t, a \sin t)-\{p\}) \rightarrow S^{*} \subset S^{1}$, and the folding of $((a \cos t, a \sin t)-\{p\})$ into itself is $f:((a \cos t, a \sin t)-\{p\}) \rightarrow((a \cos t, a \sin t)-\{p\}))$. Then there are commutative diagrams between retraction and folding such that:

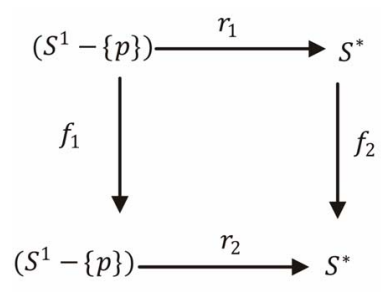

Proof. Let a retraction

$r_{1}:((a \cos t, a \sin t)-\{p\}) \rightarrow S^{*} \subset S^{1}$, be a retraction of $((a \cos t, a \sin t)-\{p\})$ into $S^{*} \subset S^{1}$. Also, let the folding $f: S^{1} \subset \mathbb{R}^{2} \rightarrow S^{1} \subset \mathbb{R}^{2}$ is given by $f_{1}:\left(S^{1}-\{p\}\right) \rightarrow\left(S^{1}-\{p\}\right)$ and $f_{2}: S^{*} \rightarrow S^{*}$, also, $r_{2}:\left(S^{1}-\{p\}\right) \rightarrow S^{*}$ such that

$$
f_{2}{ }^{\circ} r_{1}=r_{2}^{\circ} f_{1}
$$

Let $C=\bigcup_{i \in \mathbb{Z}_{+} \cup \mathbb{Z}_{-}} S^{1}\left(\left(\frac{1}{i}, 0\right), \frac{1}{i}\right) \subset \mathbb{R}^{2}$ where

$\mathrm{S}^{1}(\mathrm{G}, 1) \cong \mathrm{S}^{1}$ is the circle with center $\mathrm{G}$ and radius 1 . The intersection of all circles is denoted by 0 . Let $r_{i}: r_{i}:(C-n) \rightarrow \bar{C}, \bar{C} \subset C, n \in C$, be the retraction map of $(C-n)$ such that

$r_{1}(C-n)=\bigcup_{i=c^{2}} S^{1}\left(\left(\frac{1}{i}, 0\right), \frac{1}{i}\right), c \in \mathbb{Z}_{+} \cup \mathbb{Z}_{-}$,

$r_{2}(C-n)=\bigcup_{i=c^{2}+1} S^{1}\left(\left(\frac{1}{i}, 0\right), \frac{1}{i}\right), c \in \mathbb{Z}_{+} \cup \mathbb{Z}_{-}$,

$r_{3}(C-n)=\bigcup_{d+1<i} S^{1}\left(\left(\frac{1}{i}, 0\right), \frac{1}{i}\right), d, i \in \mathbb{Z}_{+}$,

$r_{4}(C-n)=\bigcup_{a<i<b} S^{1}\left(\left(\frac{1}{i}, 0\right), \frac{1}{i}\right), a, b, i \in \mathbb{Z}_{+} \cup \mathbb{Z}, a<b$,

$r_{5}(C-n)=S^{1}\left(\left(\frac{1}{a}, 0\right), \frac{1}{a}\right), 1<a<\infty, a \in \mathbb{Z}_{+}$,

$r_{6}(C-n)=S^{1}\left(\left(\frac{1}{b}, 0\right), \frac{1}{b}\right),-\infty<b<-1, b \in \mathbb{Z}_{-}$

$r_{7}=(0,0,0), i \rightarrow \infty$,

$r_{8}(C-n)=S^{1}((1,0), 1)$,

$r_{9}(C-n)$,

$r_{10}(C-n)=\bigcup_{a<i<a} S^{1}\left(\left(\frac{1}{i}, 0\right), \frac{1}{i}\right), a, i \in \mathbb{Z}_{+} \cup \mathbb{Z}_{-}$,

$r_{10}(C-n)=\bigcup_{i<m} S^{1}\left(\left(\frac{1}{i}, 0\right), \frac{1}{i}\right), m, i \in \mathbb{Z}_{+} \cup \mathbb{Z}_{-}$

Hence, we can introduce the following theorems: 
Theorem 11. Any circle $S^{1}$ with center $\left(\frac{1}{i}, 0\right)$ and radius $\frac{1}{i}$, where $i \in \mathbb{Z}_{+}$, is a retract of $\left(C^{+}-m\right)=\left(\bigcup_{i \in \mathbb{Z}_{+}} S^{1}\left(\left(\frac{1}{i}, 0\right), \frac{1}{i}\right)\right)-m \subset \mathbb{R}^{2}, m \in C^{+}$.

Proof. Let $C=\bigcup_{i \in \mathbb{Z}_{+}} \cup \mathbb{Z}_{-} S^{1}\left(\left(\frac{1}{i}, 0\right), \frac{1}{i}\right) \subset \mathbb{R}^{2}$ and $C^{*}$ is a retract of $(C-n), n \in C$, where $r(C-n)=C^{*}=\bigcup_{1<i} S^{1}\left(\left(\frac{1}{i}, 0\right), \frac{1}{i}\right), i \in \mathbb{Z}_{+}$. Also, If $i_{1}:\left(C^{*}-e\right) \rightarrow(C-n), e \in C^{*}$, is the inclusion map, the retractions of $\left(C^{*}-e\right)$ and $(C-n)$ are defined by $r_{1}:\left(C^{*}-e\right) \rightarrow S^{1} \quad$ and $\quad r_{2}:(C-n) \rightarrow\left(C^{+}-q\right), q \in C^{+}$, the inclusion map of $S^{1}$ is $i_{2}: S^{1} \rightarrow\left(C^{+}-q\right)$, and the retractions of $(C-n),\left(C^{+}-q\right)$, and $\left(C^{*}-e\right)$ are given by $r_{3}:(C-n) \rightarrow\left(C^{*}-e\right), r_{4}:\left(C^{+}-q\right) \rightarrow S^{1}$ and $r_{5}:\left(C^{*}-e\right) \rightarrow S^{1}$. Hence, the following diagram is commutative:

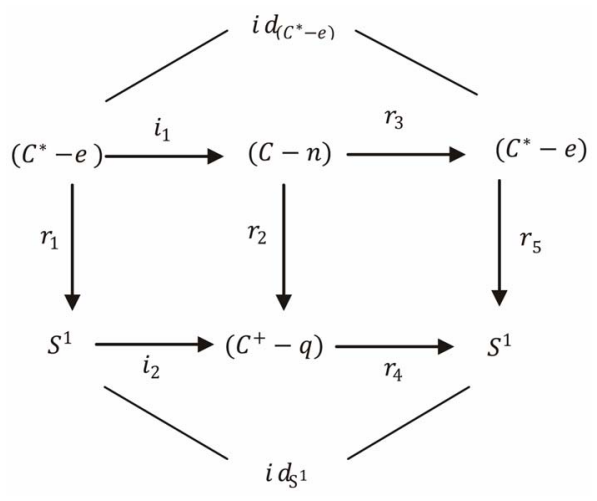

Proposition 11. Any circle $S^{1}$ with center $\left(\frac{1}{i}, 0\right)$ and radius $\frac{1}{i}$, where $i \in \mathbb{Z}_{-}$, is a retract of $\left(C^{-}-p\right)=\left(\left(\bigcup_{i \in \mathbb{Z}_{-}} S^{1}\left(\left(\frac{1}{i}, 0\right), \frac{1}{i}\right)\right)-p\right) \subset \mathbb{R}^{2}, p \in C^{-}$.

Theorem 12. Any circle $S^{1}$ with center $\left(\frac{1}{i}, 0\right)$ and radius $\frac{1}{i}$, where $i \in \mathbb{Z}_{+} \cup \mathbb{Z}_{-}$, is a retract of $(C-n)=\left(\left(\bigcup_{i \in \mathbb{Z}_{+} \cup \mathbb{Z}_{-}} S^{1}\left(\left(\frac{1}{i}, 0\right), \frac{1}{i}\right)\right)-n\right) \subset \mathbb{R}^{2}, n \in C$.
Proof. Since $\left(C^{+}-m\right), m \in C^{+}$is a retract of $(C-n)$ and any circle $S^{1}$ in $\mathbb{R}^{2}$ with center $\left(\frac{1}{i}, 0\right)$ and radius $\frac{1}{i}$, where $i \in \mathbb{Z}_{+}$, is a retract of $\left(C^{+}-m\right)$, then any circle $S^{1}$ with center $\left(\frac{1}{i}, 0\right)$ and radius $\frac{1}{i}$, where $i \in \mathbb{Z}_{+}$, is a retract of $(C-n)$. Also, Since $\left(C^{-}-p\right)$, $p \in C^{-}$is a retract of $(C-n)$ and any circle $S^{1}$ with center $\left(\frac{1}{i}, 0\right)$ and radius $\frac{1}{i}$, where $i \in \mathbb{Z}_{-}$, is a retract of $\left(C^{-}-p\right)$, then any circle $S^{1}$ with center $\left(\frac{1}{i}, 0\right)$ and radius $\frac{1}{i}$, were $i \in \mathbb{Z}_{-}$, is a retract of $(C-n)$.

Theorem 13. Any retract of circle $\left(S^{1}-m\right), m \in S^{1}$ in $\mathbb{R}^{2}$ with center $\left(\frac{1}{i}, 0\right)$ and radius $\frac{1}{i}$, where $i \in \mathbb{Z}_{+} \cup \mathbb{Z}_{-}$, is a retract of $(C-n), n \in C$.

Proof. Let $S^{1^{*}}$ is a retract of $\left(S^{1}-m\right)$, then there is a continuous map $r_{1}:\left(S^{1}-m\right) \rightarrow S^{1^{*}}, S^{1^{*}} \subset\left(S^{1}-m\right)$, where $r_{1}(x)=x, \forall x \in S^{1^{*}}$. Then the circle $\left(S^{1}-m\right)$ in $\mathbb{R}^{2}$ with center $\left(\frac{1}{i}, 0\right)$ and radius $\frac{1}{i}$, where $i \in \mathbb{Z}_{+} \cup \mathbb{Z}_{-}$, is a retract of $(C-n)$, then there is a continuous map $r_{2}:(C-n) \rightarrow\left(S^{1}-m\right)$, $\left(S^{1}-m\right) \subset(C-n)$, where $r_{2}(y)=y, \forall y \in\left(S^{1}-m\right)$. Then, $r_{1} \circ r_{2}$ is a continuous map. Also, $r_{1} \circ r_{2}(z)=z, \forall z \in S^{1^{*}} \subset\left(S^{1}-m\right)$. Then any retract of circle $\left(S^{1}-m\right)$ in with center $\left(\frac{1}{i}, 0\right)$ and radius $\frac{1}{i}$, where $i \in \mathbb{Z}_{+} \cup \mathbb{Z}_{-}$, is a retract of $(C-n)$.

\section{Conclusion}

In this paper we achieved the approval of the important of the curves in the Euclidean space by using some geometrical transformations. The relations between folding, retractions, deformation retracts, limits of folding and limits of retractions of the curves in the Euclidean space are discussed. New types of minimial retractions on curves in the Euclidean space are deduced.

\section{REFERENCES}

[1] A. E. El-Ahmady, "The Variation of the Density Functions on Chaotic Spheres in Chaotic Space-Like Minkowski Space Time," Chaos, Solitons and Fractals, Vol. 
31, No. 5, 2007, pp. 1272-1278.

doi:10.1016/j.chaos.2005.10.112

[2] A. E. El-Ahmady, "Folding of Fuzzy Hypertori and Their Retractions," Proc. Math. Phys. Soc. Egypt, Vol. 85, No. 1, 2007, pp. 1-10.

[3] A. E. El-Ahmady, "Limits of Fuzzy Retractions of Fuzzy Hyperspheres and Their Foldings," Tamkang Journal of Mathematics, Vol. 37, No. 1, 2006, pp. 47-55.

[4] A. E. El-Ahmady, "Retraction of Chaotic Black Hole," The Journal of Fuzzy Mthematics, Vol. 19, No. 4, 2011, pp. 833-838.

[5] A. E. El-Ahmady, "Fuzzy Folding of Fuzzy Horocycle," Circolo Matematico di Palermo Serie II, Tomo L III, 2004, pp. 443-450. doi:10.1007/BF02875737

[6] A. E. El-Ahmady, "Fuzzy Lobachevskian Space and Its Folding," The Journal of Fuzzy Mathematics, Vol. 12, No. 2, 2004, pp. 609-614.

[7] A. E. El-Ahmady, "The Deformation Retract and Topological Folding of Buchdahi Space," Periodica Mathematica Hungarica, Vol. 28, No. 1, 1994, pp. 19-30. doi:10.1007/BF01876366

[8] A. E. El-Ahmady and H. Rafat, "Retraction of Chaotic Ricci Space," Chaos, Solutions and Fractals, Vol. 41, 2009, pp. 394-400. doi:10.1016/j.chaos.2008.01.010
[9] A. E. El-Ahmady and H. Rafat, "A Calculation of Geodesics in Chaotic Flat Space and Its Folding," Chaos, Solutions and Fractals, Vol. 30, 2006, pp. 836-844. doi:10.1016/j.chaos.2005.05.033

[10] A. E. El-Ahmady and H. M. Shamara, "Fuzzy Deformation Retract of Fuzzy Horospheres," Indian Journal of Pure and Applied Mathematics, Vol. 32, No. 10, 2001, pp. 1501-1506.

[11] A. E. El-Ahmady and A. El-Araby, "On Fuzzy Spheres in Fuzzy Minkowski Space," Nuovo Cimento, Vol. 125B, 2010.

[12] M. Arkowitz, "Introduction to Homotopy Theory," Springer-Village, New York, 2011.

[13] G. L. Naber, "Topology, Geometry and Gauge Fields," Foundations, Springer, New York, 2011.

[14] G. L. Naber, "Topology, Geometry and Gauge Fields," Interactions, Springer, New York, 2011.

[15] M. Reid and B. Szendroi, "Topology and Geometry," Cambridge, New York, 2005. doi:10.1017/CBO9780511807510

[16] P. 1. Shick, "Topology: Point-Set and Geometry," Wiley, New York, 2007. doi:10.1002/9781118031582

[17] J. Strom, "Modern Classical Homotopy Theory," American Mathematical Society, 2011. 